\title{
Produção Agroindustrial e Sustentabilidade: um estudo de caso sobre a companhia sucroenergética São Martinho
}

\author{
Agroindustrial Production and Sustainability: a case study on the sugar-energy company São \\ Martinho
}

Recebido: 20/10/2021 | Revisado: 25/10/2021 | Aceito: 29/10/2021 | Publicado: 31/10/2021

\author{
Linnek Keven Araújo Policarpo \\ ORCID: https://orcid.org/0000-0001-7275-4328 \\ Universidade Federal de Campina Grande, Brasil \\ E-mail: linnekagroecon@gmail.com \\ Isabel Lausanne Fontgalland \\ ORCID: https://orcid.org/0000-0002-0087-2840 \\ Universidade Federal de Campina Grande, Brasil \\ E-mail: isabelfontgalland@gmail.com
}

\begin{abstract}
Resumo
A companhia sucroenergética São Martinho, fundada por italianos imigrantes, atua com quatro usinas em operação, localizadas nos estados de São Paulo e Goiás. Ambos os estados ocupam a primeira e a segunda colocação, respectivamente, no ranking nacional dos estados produtores de cana-de-açúcar, insumo utilizado para a produção de açúcar, etanol e bioenergia. A companhia São Martinho é referência no setor e garante posição entre os maiores grupos sucroenergéticos do Brasil, produzindo Açúcar, Etanol, Energia Elétrica e Subprodutos do processo de produção de açúcar e etanol, posição essa que está diretamente relacionada com o seu processo produtivo, os investimentos em inovação e o empenho em promover a sustentabilidade. O presente estudo de caso trata da produção e sustentabilidade da companhia na ótica da economia industrial.
\end{abstract}

Palavras-chave: Cana-de-açúcar; Usina sucroenergética; São Martinho; Economia.

\begin{abstract}
The São Martinho sugar-energy company, founded by Italian immigrants, has four plants in operation, located in the states of São Paulo and Goiás. Both states rank first and second, respectively, in the national ranking of sugarcane producing states, the input used for the production of sugar, ethanol and bioenergy. The São Martinho company is a reference in the sector and assures a position among the largest sugar-energy groups in Brazil, producing Sugar, Ethanol, Electric Energy and By-products of the sugar and ethanol production process. This position is directly related to its production process, investments in innovation, and commitment to promote sustainability. This case study deals with the company's production and sustainability from an industrial economy perspective.
\end{abstract}

Keywords: Sugarcane; Sugar-Energy Plant; São Martinho; Economy.

\section{Introdução}

Historicamente, a companhia São Martinho foi fundada por italianos. No final do século XIX a família Ometto imigrou para o Brasil. E a partir de então, o primeiro engenho de cana-de-açúcar foi montado em 1914, no sítio Olaria. Na Fazenda Boa Vista, região de Limeira, foi produzido açúcar pela primeira vez em 1932. Em 1937, a Usina Iracema foi adquirida e transformada em uma destilaria de álcool, passando a fabricar açúcar também em 1946. Em 1949, foi adquirida a Usina São Martinho, situada na cidade de Pradópolis, se transformando em uma das maiores processadoras de cana do mundo (São Martinho, 2021d). 
Figura 1 - Família Ometto.

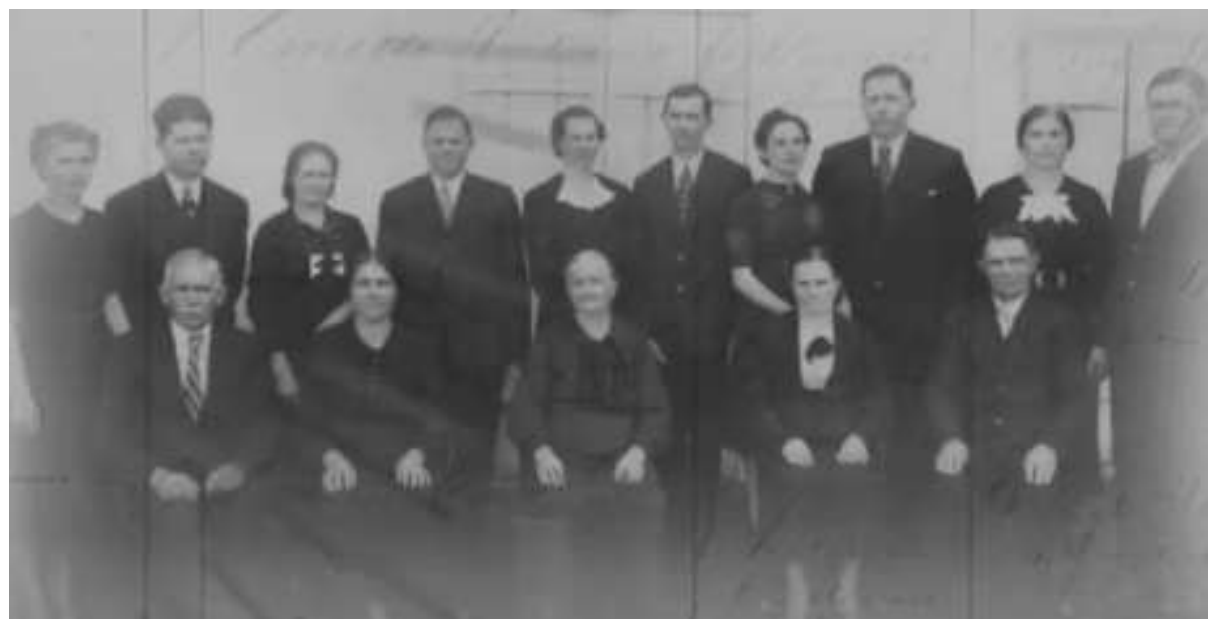

Fonte: Vídeo institucional - São Martinho.

Desde sempre as usinas foram evoluindo. Segundo a própria companhia, São Martinho foi consolidada como grupo empresarial com a padronização de uma marca nos últimos anos, o que acabou aprimorando o fortalecimento do seu desempenho. Conforme Kupfer e Hasenclever (2013), “os produtos são diferenciados segundo diversos aspectos como: local da oferta, qualidade do produto ou percepção da marca” (p. 67).

Figura 2 - Marca da Companhia.

\section{W' São Martinho}

Fonte: Site da companhia São Martinho.

A companhia sucroenergética opera com quatro (4) usinas, sendo elas: Usina São Martinho, em Pradópolis, na região de Ribeirão Preto - SP, Usina Iracema, em Iracemápolis, na região de Limeira - SP; Usina Santa Cruz, localizada em Américo Brasiliense - SP e Usina Boa Vista, em Quirinópolis - GO. Segundo o IMB (2018), o estado Goiás é o $2^{\circ}$ maior produtor nacional de cana-de-açúcar, e o $2^{\circ}$ maior produtor nacional de etanol. Os dados do IBGE (2017) mostram o estado de São Paulo como o maior produtor de cana-de-açúcar. 
Figura 3 - Usinas da Companhia.
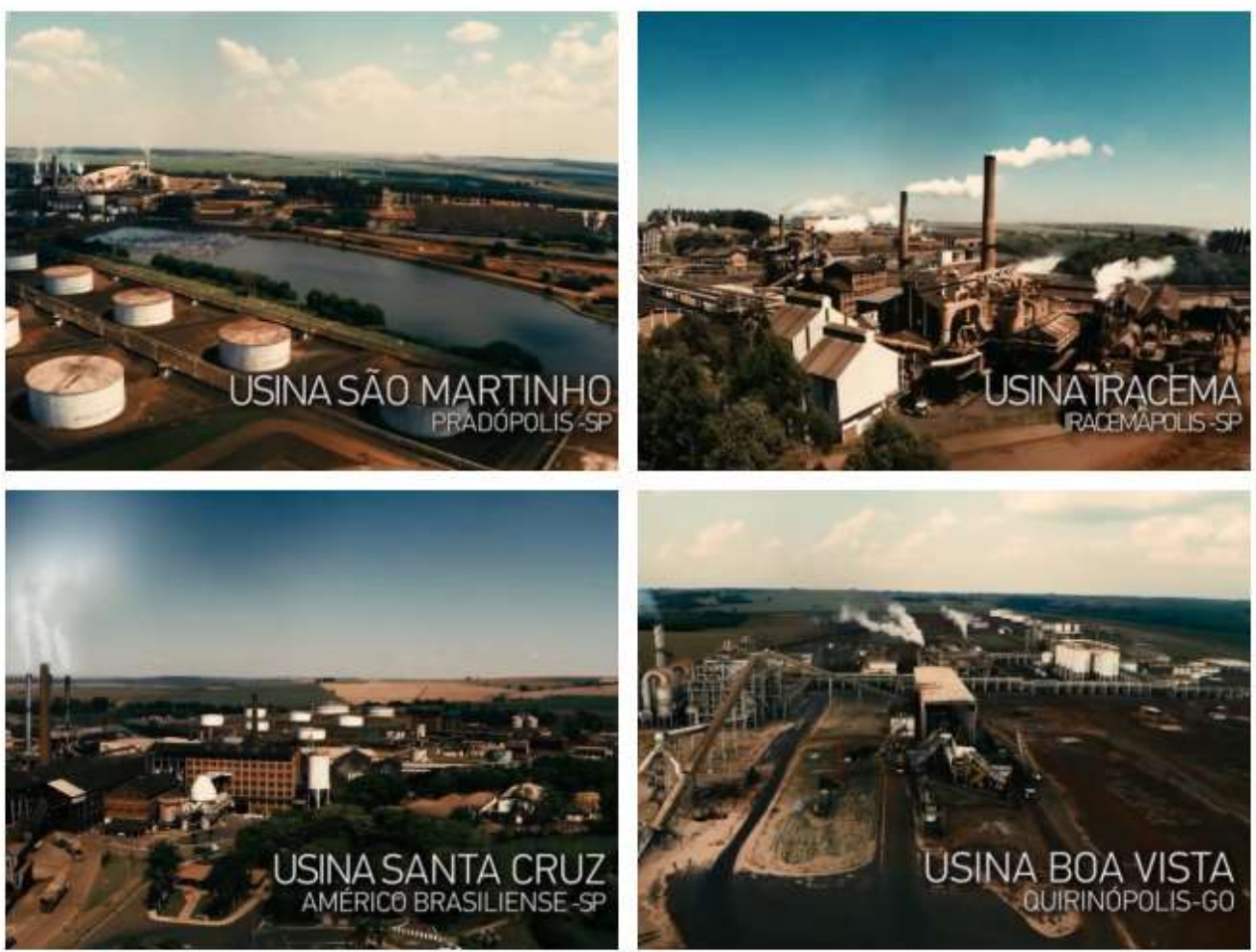

Fonte: Vídeo institucional - São Martinho (2021h).

O referente estudo de caso irá tratar da produção e a sustentabilidade sob a ótica da economia industrial na companhia São Martinho, bem como o impacto para o desenvolvimento da companhia agroindustrial, que é referência no setor do agronegócio sendo um dos maiores grupos sucroenergéticos brasileiros.

\section{Metodologia}

Segundo Bocchi et al. (2004), o estudo de caso se constitui em uma análise acerca de um determinado objeto, considerando a totalidade da qual faz parte. Dentre os métodos de procedimento adotados na pesquisa das ciências econômicas, o presente estudo foi elaborado utilizando o estudo de caso como método de procedimento. Portanto, o estudo de caso em questão é voltado para o estudo da companhia sucroenergética São Martinho, para investigar e descrever o desenvolvimento da sua produção, bem como as características de sua atuação no mercado e a questão da sustentabilidade.

\section{Resultados e Discussão}

\subsection{Produção e diversificação de produtos}

A companhia São Martinho produz Açúcar, Etanol, Energia Elétrica e Subprodutos do processo de produção de açúcar e etanol. Com quatro usinas operando, São Martinho conta com 12,5 mil colaboradores. Apresenta uma área agrícola de colheita de 350.000 hectares, onde a colheita é completamente mecanizada, e uma capacidade de moagem de 24 milhões de toneladas de cana-de-açúcar. Sua capacidade de estocagem de açúcar é de 820 mil toneladas, enquanto sua capacidade de estocagem de etanol é de 740 mil m³. Sua flexibilidade de produção entre açúcar e etanol é de $35 \%$ para o primeiro e $65 \%$ para o segundo (São Martinho, 2021e). 
Figura 4 - Açúcar, Etanol, Energia Elétrica e Cana processada por safra.

\begin{tabular}{|c|c|c|c|c|c|c|c|c|c|c|}
\hline $\begin{array}{c}\text { Cana } \\
\text { Processada } \\
\text { (milhões } \\
\text { de tons) }\end{array}$ & 13,1 & 11,4 & 12,9 & 15,6 & 18,7 & 20,0 & 19,3 & 22,2 & 20,5 & 22,6 \\
\hline $\begin{array}{l}\text { Açúcar } \\
\text { (mil tons) }\end{array}$ & 873 & 774 & 969 & 986 & 1.231 & 1.230 & 1.301 & 1.407 & 992 & 1.106 \\
\hline $\begin{array}{c}\text { Etanol (mil } \\
m^{3} \text { ) }\end{array}$ & 565 & 448 & 451 & 640 & 791 & 750 & 667 & 953 & 1.097 & 1.172 \\
\hline
\end{tabular}

Fonte: San Martinho (2021).

A safra 2019/2020 destaca-se, segundo os dados do Relatório Anual e de Sustentabilidade São Martinho (2019/2020), mostrando que a companhia obteve receita líquida de $\mathrm{R} \$ 3,7$ bilhões e um lucro líquido de R \$639 milhões na safra. Os valores menores dos indicadores de produção da safra 2018/2019 são justificados pela queda da produtividade resultado da forte estiagem observada ao longo de toda a safra.

Segundo Kupfer e Hasenclever (2013), operar com a diversificação de produtos possibilita a redução dos custos médios. A existência de fatores comuns, a existência de reserva de capacidade, e as complementaridades tecnológicas e comerciais são três fontes de economia de escopo. Havendo economia de escala e escopo concomitantemente na cadeia produtiva, observa-se a obtenção de ganhos técnico-produtivos. Dessa forma, os geradores de energia adquiridos pelas usinas sucroenergéticas servem como um fator comum à produção do portifólio de produtos da companhia. A cana-de-açúcar é insumo comum para produzir o açúcar, o etanol e a energia.

São produzidos vários tipos de açúcar bruto, como o do tipo branco. Nos últimos anos, o principal deles tem sido o do tipo VVHP (Very Very High Polarization), um tipo de açúcar padrão negociado no mercado internacional. O processo inicia-se desde a produção agrícola com a plantação da cana-de-açúcar e seu gerenciamento, em seguida a cana é colhida de forma $100 \%$ mecanizada e transportada até chegar na usina. A cana-de-açúcar entra no processo de moagem para que o caldo seja extraído para ser fabricado o açúcar e o etanol, e o bagaço é direcionado para a produção de energia. Concluído essa etapa, inicia-se o tratamento do caldo para retirar as impurezas e em seguida entra no processo de evaporação para obter o xarope concentrado. É realizado o cozimento para a obtenção da massa e após é feita a centrifugação para a obtenção do açúcar. Do açúcar centrifugado obtém-se como subproduto o melaço. Por fim, o açúcar entra no processo de secagem. Após todas essas etapas, o açúcar é comercializado (São Martinho, 2021a).

Em relação à produção de etanol, após obtido o caldo no processo de moagem, ocorre o tratamento para a remoção de impurezas. Em seguida o caldo tratado recebe leveduras para que aconteça a fermentação, originando o vinho fermentado. O vinho é centrifugado, o que separa a levedura, para o próximo ciclo de fermentação, do etanol e da água. No processo de destilação se obtém o etanol hidratado. Com a desidratação, se obtém o etanol anidro (São Martinho, 2021c).

Sobre a produção de energia, com o processo de moagem para a produção de açúcar e etanol, obtém-se o bagaço da cana que é enviado para alimentar as caldeiras das usinas. Aqui é o bagaço é queimado gerando vapor. Esse vapor é transformado em energia térmica, energia mecânica e energia elétrica. A energia elétrica é usada pelas usinas e vendido o excedente (São Martinho, 2021b). 
Como subprodutos do processo de produção de açúcar e etanol, a São Martinho fabrica e comercializa também levedura, óleo fúsel e etanol amílico puro. A levedura da fermentação da cana de açúcar no processo de produção nas usinas sucroalcooleiras pode ser posteriormente recuperada e seca para ser destinada à alimentação animal. O óleo fúsel, utilizado como solvente, por exemplo, é constituído por uma mistura de álcoois superiores obtida nas várias etapas de purificação do etanol. Os subprodutos servirão de insumos para outras atividades produtivas.

A companhia tem investido ao longo do tempo, em melhorias de processos, mudanças tecnológicas que melhoram sua eficiência operacional. A automação agrícola 4.0, com a gestão da operação agrícola em tempo real, de maneira remota, a utilização de ferramentas como inteligência artificial (AI) e Advanced Analytics, os processos e as performances dos equipamentos são otimizados. Outro exemplo de inovação se refere ao plantio com o método de MPB (Mudas Pré-Brotadas) atrelado à MEIOSI (Método Inter-rotacional Ocorrendo Simultaneamente), que permite para 1 hectare de mudas de cana-deaçúcar 560 hectares plantados, o que no sistema convencional era apenas 4 hectares plantados.

\subsection{Sustentabilidade}

Como compromisso com o meio ambiente, colaboradores, clientes e consumidores, fornecedores e a comunidade a qual a São Martinho abrange, a sustentabilidade é estrategicamente essencial na companhia. É feito a reutilização dos resíduos oriundos do processo de produção. A vinhaça, resíduo do processo de produção do etanol, é utilizado para a fertirrigação do canavial. A torta de filtro, resíduo da clarificação do caldo de cana, é utilizado para formar um composto usado no plantio substituindo fertilizantes minerais. Faz a gestão do uso racional da água investindo em projetos com essa finalidade.

Promove projetos ambientais em termos de preservação e educação, tais como: Centro de Educação Ambiental (CEA), realizando trabalhos de conscientização para sociedade; Viveiro de Mudas, uma iniciativa para o reflorestamento e para doação para comunidade; Viva a Natureza, uma iniciativa de preservação do meio ambiente com mais de 4 milhões de mudas de árvores plantadas; Cartilha de prevenção contra incêndios, que é voltado para colaboradores e as comunidades regionais próximas (São Martinho, 2021g).

De acordo com o Relatório Anual e de Sustentabilidade São Martinho (2019/2020), é a primeira empresa produtora de etanol a obter certificação para emitir e comercializar créditos de descarbonização (CBios), no âmbito do RenovaBio. Mais além, as usinas possuem outras certificações importantes. 
Figura 5 - Certificações das Usinas da Companhia.

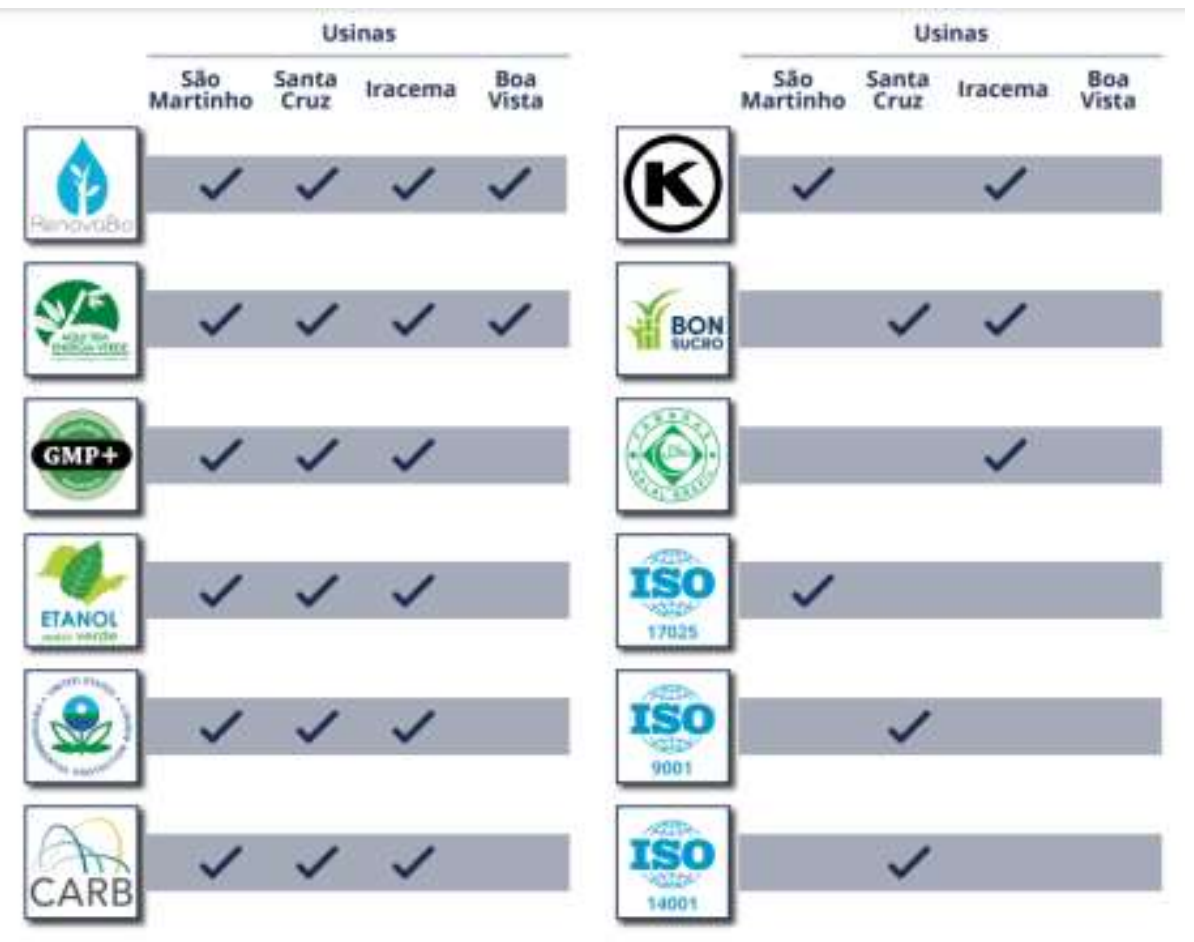

Fonte: Relatório Anual e de Sustentabilidade 2019/20 - São Martinho.

\section{Considerações Finais}

A diversificação de produtos da companhia resulta na redução dos custos, dado que o custo rm produzir produtos conjuntamente é menor do que o custo de produzi-los separadamente. Com um mix de produtos arrojado, a estratégia de especialização produtiva, dá à empresa uma maior segurança, reduzindo assim a variância do comportamento das suas receitas, diminuindo, portanto, o risco de operações em mercados diferentes, seja do açúcar, do etanol ou energia. As inovações ocorridas no plantio com os métodos MPB+MEIOSI, em substituição ao sistema convencional, afetou em grande proporção a produtividade do canavial de maneira positiva.

A usina de Iracema da companhia São Martinho possui certificação Fambras Halal Brazil, o que garante produtos lícitos, seguros para a comunidade muçulmana mundial de acordo com requisitos legais e critérios determinados pela jurisprudência islâmica. Assim, a possibilita um market share do comércio Halal, tornando-a mais relevante diante dos competidores do setor agroindustrial em que atua.

Todo o processo produtivo, desde o plantio até a obtenção do produto final, juntamente com os investimentos em inovações tecnológicas realizadas, seja de processo ou de produto, e o empenho garantidor da sustentabilidade que a São Martinho realiza, a torna referência no setor e garante posição entre os maiores grupos sucroenergéticos do Brasil. A companhia tem potencial para enfrentar as intempéries do mercado.

Sugere-se para trabalhos futuros, a avaliação dos investimentos em P\&D, na área agrícola, a fim de aumentar o potencial produtivo da cana-de-açúcar a patamares de nível ótimo, na área industrial a fim de que as inovações tecnológicas utilizadas no processamento do insumo possam cada vez mais proporcionar eficiência produtiva. No âmbito da sustentabilidade, as usinas da companhia se adequem aos requisitos para alcançar as certificações que ainda não obtiveram, principalmente a usina Boa Vista. 


\section{Referências}

Bocchi, J. I., Borba, J. T., Agüero, P. H. V. \& Macedo, Z. L. (2004). Monografia para economia. Saraiva.

Britto, J. (2013). Cooperação Interindustrial e Redes de Empresas. In: Kupfer, D.; Hasenclever, L (orgs.). Economia industrial: Fundamentos teóricos e práticas no Brasil. (2a ed.), Elsevier.

Fambras Halal. (2021). Como Certificar. http://halal.org.br/como-certificar.

Ferreira, J. M., Jaguaribe, D. C. A. \& Silva, R. S. (2020). Estudo da arte sobre a utilização do óleo fúsel a partir da produção de etanol da cana-de-açúcar. In: Ribeiro, J. C. (org). Ciências Exatas e da Terra: Conhecimentos Estratégicos para o Desenvolvimento do País. Ponta Grossa, PR: Ed. Atena. https://www.atenaeditora.com.br/post-artigo/37411.

Instituto Brasileiro de Geografia e Estatística (IBGE). (2017). Censo Agro 2017. https://censos.ibge.gov.br/agro/2017/templates/censo _agro/resultadosagro/agricultura.html?localidade $=0 \&$ tema $=76434$.

Instituto Mauro Borges de Estatísticas e Estudos Socioeconômicos (2018). Goiás - Visão Geral. https://www.imb.go.gov.br/ind ex.php?option=com_content $\&$ view=article\&id=79\&Itemid=145.

Losekann, L. \& Gutierrez, M. (2013). Diferenciação de Produtos. In: Kupfer, D. \& Hasenclever, L (orgs.). Economia industrial: Fundamentos teóricos e práticas no Brasil. 2ed. Rio de Janeiro: Elsevier. pag. 67.

São Martinho. (2019). Relatório Anual e de Sustentabilidade 2018/19. https://www.saomartinho.com.br/Download.aspx?Arquivo=qANN7 yZHF6C1Wzr7w84Prw==\&IdCanal=E/5CI5hbGiTfjZ7mhu2y5w==.

São Martinho. (2020). Relatório Anual e de Sustentabilidade 2019/20. https://www.saomartinho.com.br/Download.aspx?Arquivo=pfv uAA7Y9+cJ2N7rhlMTfw==\&IdCanal=E/5CI5hbGiTfjZ7mhu2y5w==.

São Martinho. (2021a). Açúcar. https://www.saomartinho.com.br/show.aspx?idCanal=DzWqSf3TRtybh40H4yX3JQ==.

São Martinho. (2021b). Energia Elétrica. https://www.saomartinho.com.br/show.aspx?idCanal=P0WoVzcsvgejc/8M8keoEA==.

São Martinho. (2021c). Etanol. https://www.saomartinho.com.br/show.aspx?idCanal=rhw93OXFh1cptbt5Xx8owA==.

São Martinho. (2021d). Institucional. https://www.saomartinho.com.br.

São Martinho. (2021e). Nossos Números. https://www.saomartinho.com.br/show.aspx?idCanal=jVGUPHOWvMeYYUV+LdgEcQ==.

São Martinho. (2021f). Prêmios \& Certificações. https://www.saomartinho.com.br/show.aspx ?idCanal=r5QVJOnmmxnToAiInIBtLA==.

São Martinho. (2021g). Projetos Ambientais. https://www.saomartinho.com.br/show.aspx?idCanal=J8na0SPKdYOYvrsmo+Ii6w==.

São Martinho. (2021h). Vídeos institucionais. https://www.saomartinho.com.br/show.aspx?idCanal=iy09uG+VkfPLeHBUpVBNTw==. 